
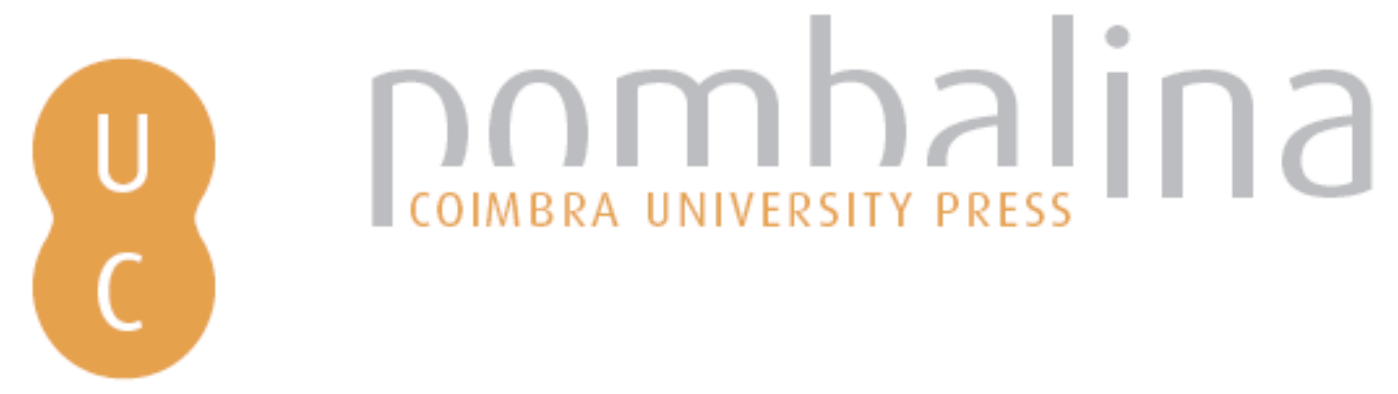

\title{
Padrão geográfico e sazonal de internamentos por perturbações mentais
}

Autor(es): $\quad$ Almendra, Ricardo; Loureiro, Adriana; Santana, Paula

Publicado por: Imprensa da Universidade de Coimbra

URL

persistente:

URI:http://hdl.handle.net/10316.2/38276

DOI:

DOI:http://dx.doi.org/10.14195/978-989-26-1105-1_2

Accessed : $\quad$ 26-Apr-2023 12:58:18

A navegação consulta e descarregamento dos títulos inseridos nas Bibliotecas Digitais UC Digitalis, UC Pombalina e UC Impactum, pressupõem a aceitação plena e sem reservas dos Termos e Condições de Uso destas Bibliotecas Digitais, disponíveis em https://digitalis.uc.pt/pt-pt/termos.

Conforme exposto nos referidos Termos e Condições de Uso, o descarregamento de títulos de acesso restrito requer uma licença válida de autorização devendo o utilizador aceder ao(s) documento(s) a partir de um endereço de IP da instituição detentora da supramencionada licença.

Ao utilizador é apenas permitido o descarregamento para uso pessoal, pelo que o emprego do(s) título(s) descarregado(s) para outro fim, designadamente comercial, carece de autorização do respetivo autor ou editor da obra.

Na medida em que todas as obras da UC Digitalis se encontram protegidas pelo Código do Direito de Autor e Direitos Conexos e demais legislação aplicável, toda a cópia, parcial ou total, deste documento, nos casos em que é legalmente admitida, deverá conter ou fazer-se acompanhar por este aviso. 


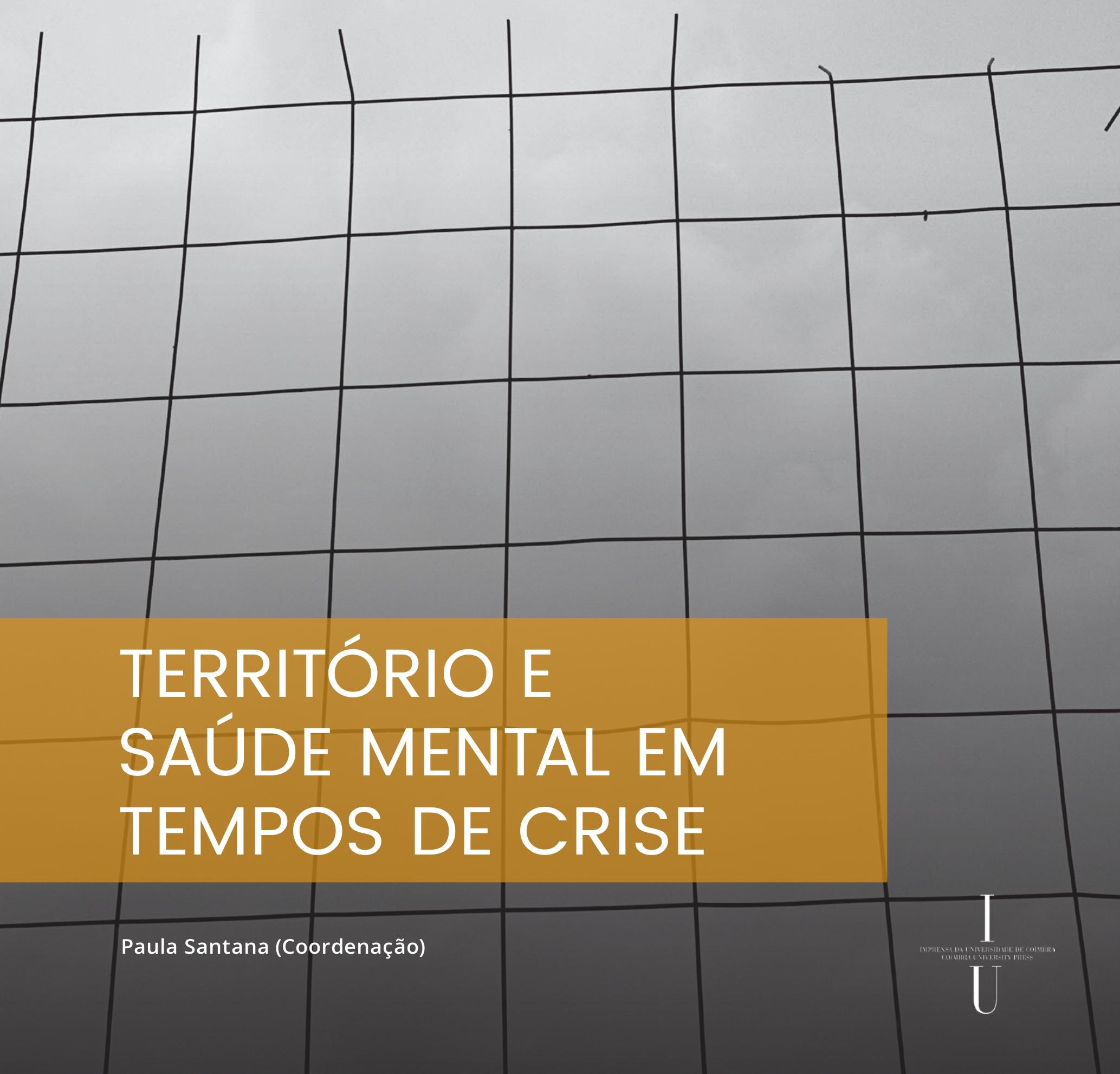




\subsection{Padrão geográfico e sazonal de internamentos por perturbações mentais}

Ricardo Almendra ${ }^{(1,2)}$, Adriana Loureiro ${ }^{(1,2)}$ e Paula Santana ${ }^{(1,2)}$

(1) Centro de Estudos de Geografia e Ordenamento do Território (CEGOT), Universidade de Coimbra;

(2) Departamento de Geografia e Turismo, Universidade de Coimbra

DOI: http://dx.doi.org/10.14195/978-989-26-1105-1_2

A incidência da doença mental apresenta variações territoriais, entre países e entre regiões do mesmo país. Apesar disso, o conhecimento sobre a distribuição geográfica da doença mental é ainda insuficiente. $O$ objetivo deste trabalho é analisar o padrão espacial e sazonal da morbilidade hospitalar por perturbações mentais em Portugal Continental, entre 2008 e 2012.

Com base na informação proveniente da base de dados dos Grupos de Diagnósticos Homogéneos (GDH) fornecida pela Administração Central do Sistema de Saúde (ACSS), foram analisados os internamentos hospitalares cuja principal causa foi perturbação mental (CID 9: 290-319) ou causa externa (principal) de suicídio e lesão auto-infligida (CID9: E95), agregando a informação para as 28 NUT III do Continente. Foram calculadas taxas de internamento padronizadas, razões padronizadas de internamento e testou-se a possível existência de aumentos sazonais estatisticamente significativos através do teste chi-square goodness of fit.

Verificou-se que a morbilidade hospitalar por perturbações mentais é superior nas mulheres, principalmente nas classes etárias mais envelhecidas. Foram identificados importantes contrastes territoriais, destacando-se a região Centro pelos valores elevados. Contrariamente à maioria das causas de internamento, e de morte, as perturbações mentais são superiores na primavera e verão e muito inferiores no inverno. A identificação de territórios de risco proporciona importantes informações que devem ser incorporadas no desenvolvimento de políticas e estratégias que resultem em melhores resultados em saúde.
The incidence of mental disease has important spatial disparities between countries and even between regions of the same country. Despite this strong spatial behavior, the knowledge about its geography is still scarce. Thus, this research aims to analyze the spatial and seasonal pattern of hospital morbidity through mental disorders in Portugal mainland between 2008 and 2012.

Hospital admissions through mental disorders (ICD 9: 290-319) or with main external cause of suicide and self-inflicted injury (ICD 9: E95), by NUT III, were analyzed. Morbidity records were provided by ACSS and population data by National Statistics. Through these data, standardized hospital admission rate and ratio were calculated and the chi-square goodness of fit teste was used to identify significant seasonal increases in morbidity. Hospital morbidity by mental disorders is higher in women, mainly in older ages. Important spatial contrasts were identified, highlighting the Center Region by the high hospitalization rates. Unlike the majority of causes of morbidity, and death, mental disorders are higher in spring and summer and lower in winter. Identifying hazardous places provides important insights that should be considered in the development of policies and strategies in order to obtain better health outcomes. 


\section{Introdução}

Os resultados do Inquérito Nacional de Saúde de 2014 indicam que em Portugal 25,4\% da população residente, com 15 ou mais anos, apresenta sintomas de depressão. Estima-se que os custos diretos e indiretos associados às perturbações mentais ascendam a cerca de $20 \%$ de todos os custos em saúde do orçamento público (DGS, 2013). A sua definição e quantificação são importantes para a comunidade académica, profissionais de saúde e políticos, particularmente na avaliação dos impactos da doença, dos resultados em saúde e na análise dos determinantes em saúde (Moriarty et al., 2009).

A doença mental está associada a diversos factores; na literatura são frequentemente referidos determinantes biológicos (e.g. sexo, idade), comportamentais (e.g. consumo de substâncias) ou relativos às condições ambientais (e.g. acesso a serviços, poluição atmosférica) (Rohrer, Rush Pierce \& Blackburn, 2005; Patel et al., 2010; Annerstedt et al., 2012). A identificação das condições associadas ao risco de doença mental é fundamental para o desenvolvimento e aplicação de medidas que visem minorar os impactos e a própria incidência da doença mental.

Apesar da importância da doença mental, em termos de custos sociais e económicos (Whiteford et al., 2013), o conhecimento sobre a sua distribuição geográfica ainda é insuficiente (Moriarty et al., 2009). Segundo Holley (1998), os primeiros estudos remontam ao final do século XIX e analisam o comportamento dos países relativamente à taxa de mortalidade por suicídio. Os estudos de Giles-Sims \& Lockhart (2006) e Hsu et al. (2015) identificam importantes diferenças entre territórios, sugerindo contrastes geográficos em termos da saúde mental da população.

Para além da variação espacial verificada na mortalidade por suicídio, sugerindo a importância das condições territoriais e de contexto, são também reportados importantes contrastes temporais e sazonais na doença mental (Ajdacic-Gross et al., 2005; Woo, Okusaga \& Postola- che, 2012) que sugerem, também, a influência das condições meteorológicas (Nogueira et al., 2009) e climáticas (Dixon \& Kalkstein, 2009).

A variação sazonal da mortalidade é uma característica partilhada por várias causas de morte (Eurowinter Group, 1997; Burkart et al., 2011; Almendra et al., 2015). Encontram-se padrões sazonais em doenças infeciosas, parasitárias, respiratórias, circulatórias, mas também em causas externas de mortalidade como o suicídio ou os acidentes rodoviários. No entanto, enquanto a maior parte das doenças do aparelho circulatório e respiratório (as principais causas de morte) apresentam um aumento de mortalidade durante os meses mais frios (Eurowinter Group, 1997; Healy, 2003; Rau, 2006) o suicídio tende a ser superior na primavera e no verão (Woo, Okusaga \& Postolache, 2012; Christodoulou et al., 2012; Roehner, 2015).

Assim, este trabalho pretende analisar o padrão espacial e sazonal da morbilidade hospitalar por perturbações mentais em Portugal Continental entre 2008 e 2012.

\section{Material e métodos}

\subsection{Dados}

Analisou-se a morbilidade hospitalar devida a perturbações mentais, utilizando os registos de internamentos hospitalares presentes na base de dados dos Grupos de Diagnósticos Homogéneos (GDH) disponibilizados pela Administração Central do Sistema de Saúde. Consideraram-se os episódios de internamento urgentes em hospitais públicos do Serviço Nacional de Saúde (SNS) de residentes no Continente, cujo registo de entrada nos serviços hospitalares se verificou no quinquénio 2008-2012. Foram extraídos os episódios de internamento com diagnóstico principal de perturbação mental (CID 9:290-319) ou com causa externa principal de suicídio e lesão auto-infligida (CID9: E95), para cada NUT III do Continente.

Os dados populacionais foram recolhidos no Instituto Nacional de Estatística para o quinquénio de 2008-2012. 


\subsection{Métodos}

Para analisar o padrão de internamentos por perturbações mentais calcularam-se Taxas de Internamento Padronizadas (TIP), segundo o método de padronização directo e utilizando a população padrão europeia. A TIP apresenta-se como uma taxa bruta de internamentos que é ajustada considerando as diferenças existentes na estrutura etária da população, através de uma população padrão. Este método de padronização permite comparar territórios com estruturas populacionais díspares (DGS, 2006).

De modo a identificar a geografia do risco de internamento calcularam-se Razões Padronizadas de Internamento (RPI) e respectivos intervalos de confiança a 95\%. De acordo com Santana (2005), o cálculo das RPI é realizado em três passos: i) determinam-se, para o Continente, as taxas de internamento para cada grupo etário, que serão as taxas de referência; ii) estimam-se os internamentos esperados para cada grupo de idades e para cada NUT, tendo em conta a taxa de referência apurada; iii) determinam-se as RPI através da relação entre os internamentos esperados e os observados. Considera-se que regiões cujo intervalo de confiança inferior é superior ao valor de referência apresentam elevado risco de internamento; caso o intervalo de confiança superior seja inferior ao nível de referência, podese afirmar que essas regiões apresentam baixo risco de internamento.

Para testar a possível existência de sazonalidade de internamentos por perturbações mentais foi analisada a sua distribuição mensal. Os registos mensais de episódios de internamentos foram normalizados de modo a obter resultados padronizados de meses de 30 dias (Pinheiro, 1990). Seguidamente, utilizou-se o teste chi-square goodness of fit para identificar a existência de aumentos sazonais estatisticamente significativos. Este método permite comparar a diferença entre os internamentos observados e os esperados, caso se verificásse uma distribuição homogénea ao longo do ano (Alcorn et al., 2013).

\section{Resultados e discussão}

\subsection{Padrão de internamentos}

Em Portugal Continental foram identificados 114.690 episódios de internamento por perturbações mentais, no quinquénio 2008-12. O número de internamentos apresenta ligeiras variações no período em estudo, sugerindo, contudo, uma tendência decrescente de 2008 a 2012 (figura 1). Verificam-se mais internamentos nas mulheres (59.872) do que nos homens (54.818), correspondendo a uma taxa de 248 e 226 int. 100.000/hab, respetivamente nos homens e mulheres.

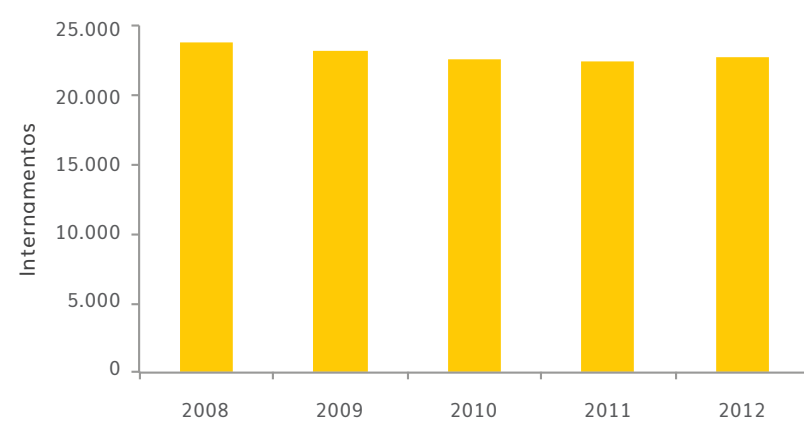

Figura 1. Internamentos por perturbações mentais por ano. Fonte: elaborado a partir da base de dados dos GDH

Verifica-se que as taxas de internamento são superiores nos homens até à classe etária dos 35 aos 39 anos, após esse grupo etário os valores são sempre superiores nas mulheres (figura 2). Tanto para homens como para mulheres, as taxas de internamentos aumentam com a idade até à classe etária dos 40 aos 44 anos, sendo o grupo que revela valores mais elevado (355 nos homens e 386 int. 100.000/hab nas mulheres). Entre os 45 e os 69 anos verifica-se uma diminuição progressiva da taxa de internamentos. Esta tendência inverte-se a partir dos 70 anos, sendo mais acentuada nas mulheres (figura 2).

A análise da TIP revela um padrão geográfico marcado por grandes disparidades, destacandose as sub-regiões da região Centro (Baixo Mondego, Pinhal Interior Norte e Sul, Beira interior Sul e Cova da Beira) com valores particularmente elevados (figura 3). Cova da Beira (a sub-região com maior TIP) regista 497 int. 100.000/hab. Em sentido oposto, o Cávado, sub-região com menor 
taxa, regista 128 int. 100.000/hab. Ou seja, os resultados observados na Cova da Beira são 290\% superiores ao que se verificou no Cávado. O valor do Continente é de 216 int. 100.000/hab.

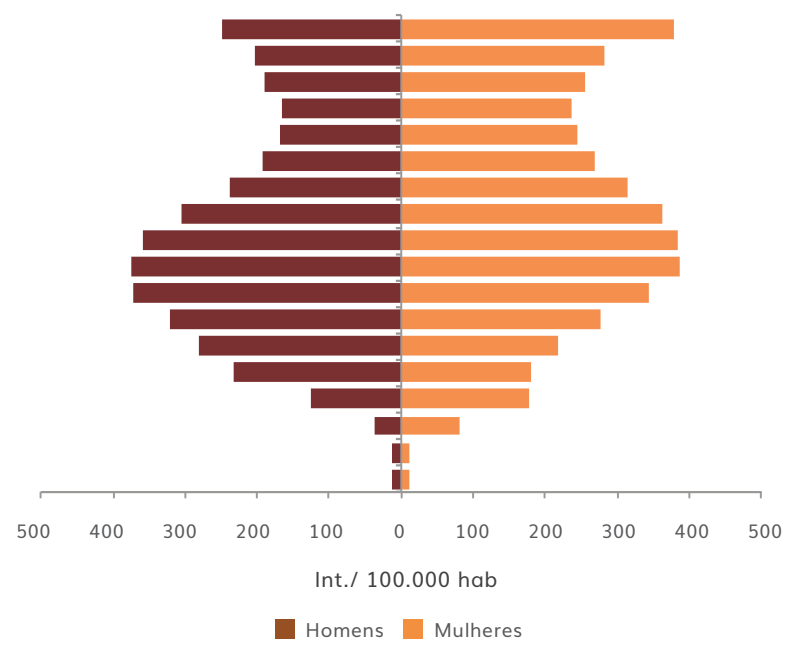

Figura 2. Taxas internamento por perturbações mentais (200812) por sexo e grupo idade.

Fonte: elaborado a partir da base de dados dos GDH

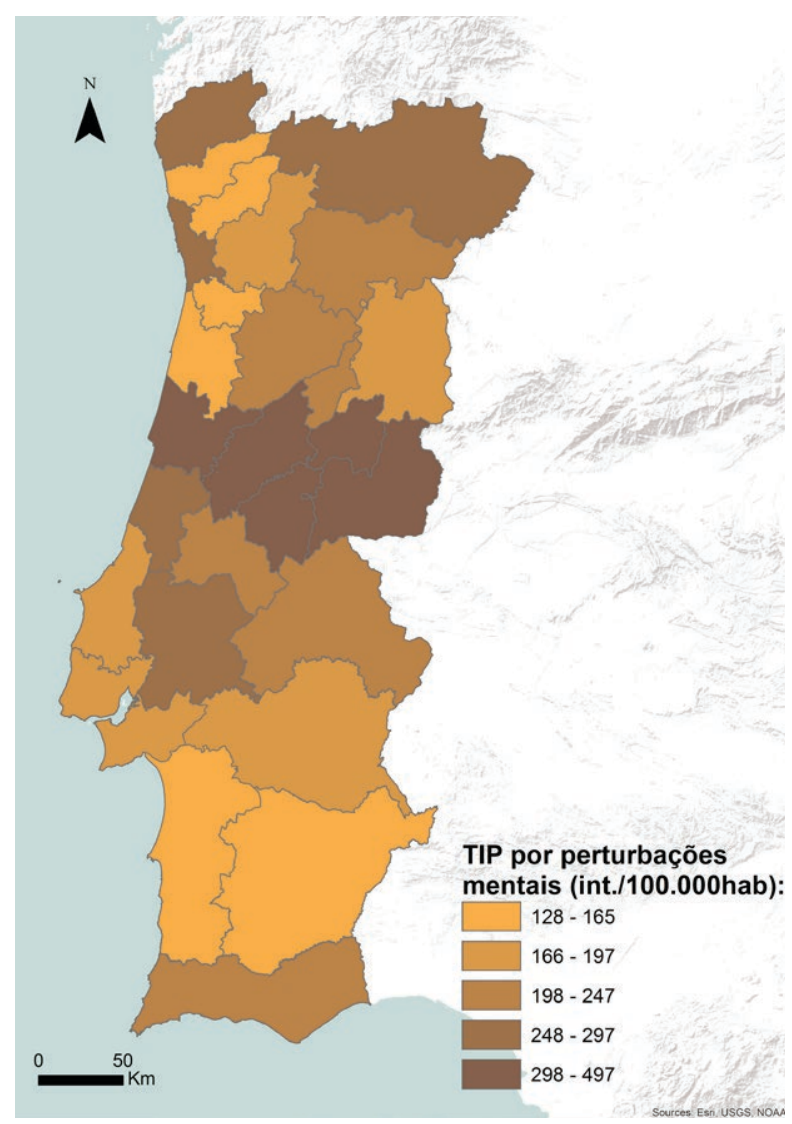

Figura 3. Taxa de internamento padronizada por perturbações mentais (2008-12).

Fonte: elaborado a partir da base de dados dos GDH

\subsection{Risco de internamento}

A análise do risco de internamento por perturbações mentais destaca a grande amplitude de resultados. Verifica-se que 15 NUT apresentam elevado risco de internamento e 11 registam significativamente menor risco que o Continente (figura 4).

A observação do padrão do Risco de Internamento (RI) coloca em evidência as NUT do Nordeste, Centro e Algarve, como regiões de elevado risco de internamento por perturbações mentais, podendo concluir-se que $39 \%$ da população reside em NUT de elevado risco. Contrariamente, $57 \%$ da população residente encontra-se em NUT de baixo risco, destacando-se o noroeste, a Área Metropolitana de Lisboa e algumas regiões do Alentejo.

Destaque para o contraste entre as NUT Grande Lisboa e Grande Porto: a primeira revela baixo risco de internamento por perturbações mentais, a segunda risco elevado.

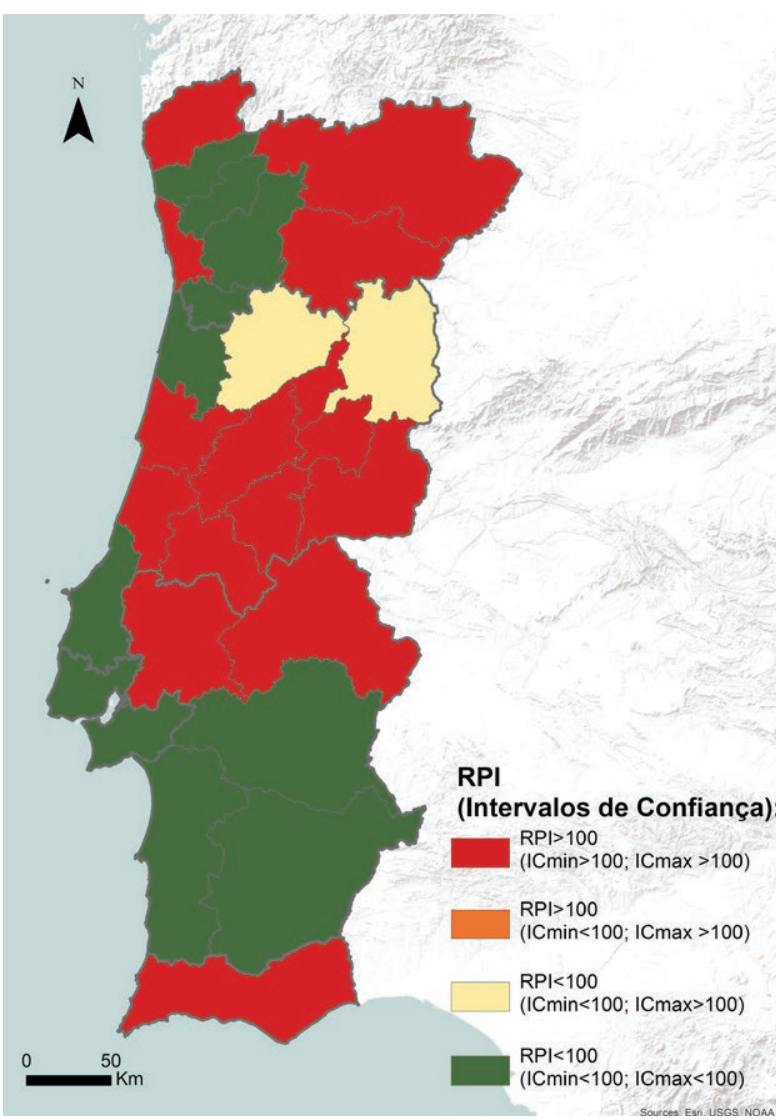

Figura 4 - Razão padronizada de internamentos por perturbações mentais (2008-12).

Fonte: elaborado a partir da base de dados dos GDH 


\subsection{Variação sazonal}

Comparando a distribuição mensal dos internamentos por perturbações mentais e por todas as causas verificam-se comportamentos distintos. Os internamentos por todas as causas aumentam durante os meses de inverno e diminuem nos meses mais quentes. Em sentido oposto, os internamentos por perturbações mentais registam os valores mais baixos (figura 5) em dezembro. De registar que esta tendência parecia atenuar-se ao longo da série temporal
Para ultrapassar a variabilidade anual (que pode resultar de fenómenos conjunturais ou de pequenos números) os internamentos foram agrupados para o quinquénio em análise (20082012). Desta forma obtêm-se dados mais estáveis, sendo possível verificar que nos meses de setembro e julho se registam significativamente mais internamentos ( $p$-value $<0,05$ ) (figura 6 ). Verifica-se a existência de diferenças entre estações ( $p$-value $<0,05$ ), sendo na primavera e no verão que se observam mais internamentos, em oposição ao inverno.

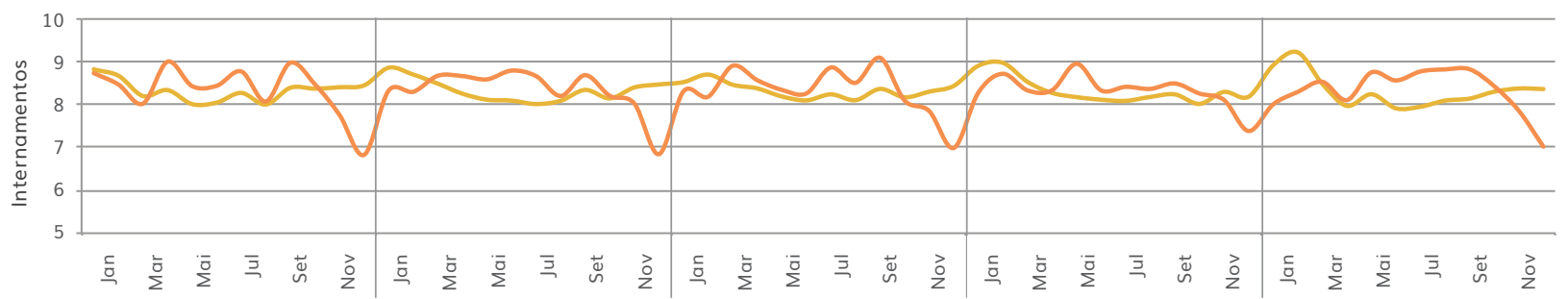

Figura 5 - Evolução da proporção mensal de internamentos por

em análise, no entanto em 2012 registaram-se valores muito semelhantes aos verificados em 2008. Verifica-se que é mais difícil identificar, claramente, o mês em que se registam mais internamentos por perturbações mentais em virtude da grande variabilidade mensal.
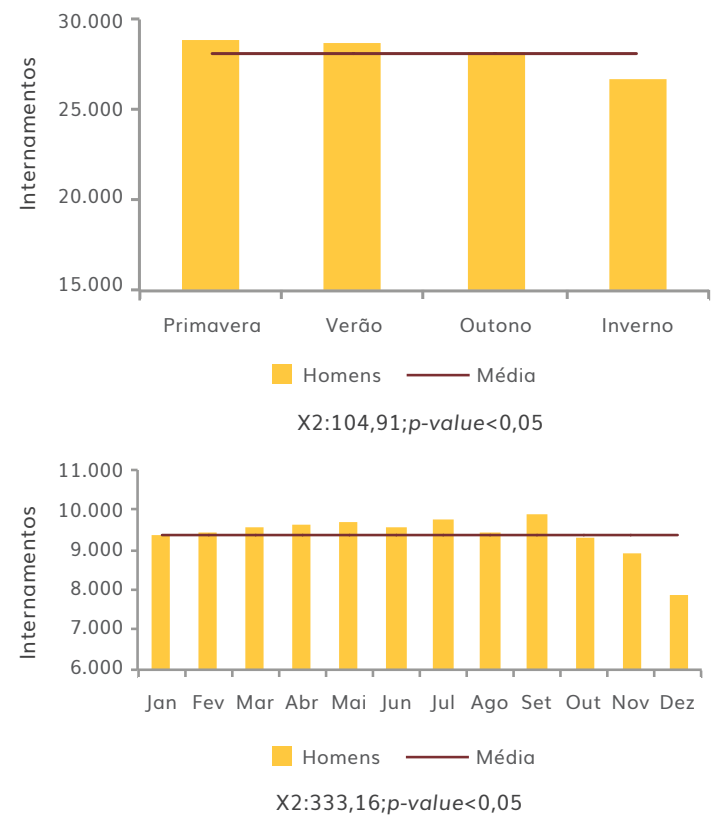

Figura 6 - Internamentos segundo os meses e estações do ano por perturbações mentais, em 2008-2012.

Fonte: elaborado a partir da base de dados dos GDH

\section{Discussão e conclusões}

A saúde mental, ou a sua ausência, é frequentemente medida através de proxys, sendo comum recorrer-se a eventos mensuráveis e concretos como o óbito ou os internamentos hospitalares por causas selecionadas (e.g. suicídio ou perturbação mental). Neste estudo foram utilizados os internamentos hospitalares por perturbações mentais ou tentativa de suicídio para analisar o padrão geográfico e sazonal da morbilidade por doença mental.

Em Portugal, a morbilidade hospitalar tem sido estuda através da base de dados dos GDH. O principal objetivo deste sistema de classificação é agrupar episódios de internamento de acordo com as características clínicas e de consumos associados de recursos, sendo a base do financiamento do internamento hospitalar. Apesar da sua riqueza, esta base de dados encontra-se sujeita a enviesamentos múltiplos que é necessário ter em conta quando se analisam os dados, principalmente quando se comparam áreas geográficas distintas. Estudos anteriores (Nicolau et al., 2009) revelaram 
que a proximidade a hospitais está associada a valores de internamento mais elevados, podendo contribuir para taxas de internamento superiores. Não deve ser desprezada a diferente disponibilidade de camas e a variação do modus operandi entre hospitais. Ressalvar ainda que esta informação se refere a episódios de internamento (não pacientes) em hospitais públicos do SNS. Apesar de não serem consideradas as unidades de saúde privadas, de acordo com um estudo Comissão Nacional para a Reestruturação dos Serviços de Saúde Mental (2008), o SNS é responsável pela maioria dos atos assistenciais e também pelo maior volume de recursos.

Foram analisados os internamentos urgentes por perturbações mentais entre 2008 e 2012, tendose verificado uma ligeira diminuição ao longo do período analisado. Estes resultados não estão de acordo com a evolução da mortalidade por suicídio, que aumentou no mesmo período: a Direção Geral de Saúde (2014) revela um aumento da taxa bruta de mortalidade e uma estagnação da taxa de mortalidade padronizada.

Verificaram-se mais episódios de internamentos nas mulheres, principalmente a partir dos 45 anos. Estes resultados estão de acordo com a literatura: Eaton et al (2012) indicam que as mulheres apresentam prevalências superiores de depressão, transtorno de ansiedade generalizada, transtorno de pânico e fobias; simultaneamente, as mulheres recorrem mais aos serviços de saúde que os homens (Velde, Boyd \& Masfety, 2011). O mesmo foi revelado pelos resultados do Inquérito Nacional de Saúde (INE \& INSA, 2015).

Observam-se variações de resultados, na TIP por perturbações mentais, entre NUT: a NUT com maior taxa é $290 \%$ superior à NUT com menor. Verifica-se, ainda, que a TIP é superior nas sub-regiões do centro do país, contrariando o padrão geográfico da mortalidade por suicídio. Santana et al (2005) e Santana et al (2015) destacam os municípios do Sul do país pela elevada mortalidade por suicídio.
Partindo da análise do risco de internamento, conclui-se existir grande variabilidade de resultados entre sub-regiões: destaque para o contraste entre a NUT Grande Lisboa e Grande Porto, sendo que o Porto apresenta piores resultados em saúde. Padrão semelhante foi encontrado por Loureiro et al (2014).

Foram encontradas importantes diferenças sazonais, reveladas através da análise da distribuição mensal e estacional dos internamentos hospitalares. O padrão sazonal destaca-se, principalmente, pelo baixo número de internamentos no inverno, particularmente em dezembro. Esta componente sazonal parecia estar a atenuar-se entre 2008 e 2011, no entanto em 2012 verificou-se novamente um grande contraste entre o número de internamentos que se verificaram no inverno e no resto do ano. Hare e Walter (1978), Clarke et al (1999) e Shapira et al (2004) identificaram resultados semelhantes relativamente à distribuição anual de internamentos por doenças mentais. Em Portugal, Monteiro e Velhas (1995) reconheceram um padrão sazonal semelhante relativo à mortalidade por suicídio.

Contrariamente ao que se verifica nas doenças do aparelho respiratório ou circulatório, onde se conhecem as consequências biológicas da exposição a temperaturas adversas, existem ainda dúvidas relativamente aos processos que justificam esta variação sazonal nas perturbações mentais (Rau, 2006; Woo, Okusaga \& Postolache, 2012). Ou seja, não existe evidência científica inequívoca sobre qual é o mecanismo bioclimático que dita a sazonalidade da doença mental (Christodoulou et al., 2012; Woo, Okusaga \& Postolache, 2012; Roehner, 2015).

A compreensão dos fatores de risco associados à variação sazonal das perturbações mentais são importantes para melhorar as estratégias preventivas e assim promover a saúde mental. Do mesmo modo, o conhecimento adquirido a partir de estudos ecológicos sobre as disparidades geográficas em saúde mental poderão ter importantes implicações para o desenvolvimento de políticas e estratégias que resultem em melhores e mais efetivos resultados em saúde. 


\section{Agradecimentos}

Este estudo foi desenvolvido no âmbito do projeto de investigação PTDC/ATP-GEO/4101/2012, SMAILE, Saúde Mental - Avaliação do Impacto das Condicionantes Locais e Económicas, e das bolsas de doutoramento SFRH/BD/92568/2013 e SFRH/BD/92369/2013, financiados por Fundos FEDER através do Programa Operacional Factores de Competitividade - COMPETE e por Fundos Nacionais através da FCT - Fundação para a Ciência e a Tecnologia.

Agradece-se ao grupo de investigação do projeto SMAILE (Benedetto Saraceno, Carla Nunes, Graça Cardoso, Joana Lima, João Ferrão, José Caldas de Almeida, Manuela Silva, Maria do Rosário Partidário e Pedro Pita Barros) pelos contributos ao longo destes dois anos de desenvolvimento do projeto.

\section{Referências Bibliográficas}

Ajdacic-Gross, V., Bopp, M., Sansossio, R., Lauber, C., Gostynski, M., Eich, D., Gutzwiller, F., Rössler, W. (2005). Diversity and change in suicide seasonality over 125 years. Journal of Epidemiology \& Community Health, 59(11), 967-972. doi:10.1136/jech.2004.030981

Alcorn, K., Gerrard, J., Macbeth, D., \& Steele, M. (2013). Seasonal variation in health care-associated bloodstream infection: increase in the incidence of gram-negative bacteremia in nonhospitalized patients during summer. American Journal of Infection Control, 41(12), 1205-8. doi:10.1016/j.ajic.2013.05.019

Almendra, R., Santana, P., Vasconcelos, J., \& Freire, E. (2015). Seasonal mortality patterns due to diseases of the circulatory system in Portugal. Geography, environment, sustainability, 1(8), 71-77.

Annerstedt, M., Ostergren, P.-O., Björk, J., Grahn, P., Skärbäck, E., \& Währborg, P. (2012). Green qualities in the neighbourhood and mental health - results from a longitudinal cohort study in Southern Sweden. BMC Public Health, 12, 337. doi:10.1186/1471-2458-12-337

Burkart, K., Khan, M. H., Krämer, A., Breitner, S., Schneider, A., \& Endlicher, W. R. (2011). Seasonal variations of all-cause and cause-specific mortality by age, gender, and socioeconomic condition in urban and rural areas of Bangladesh. International Journal for Equity in Health, 10(1), 32. doi:10.1186/1475-9276-10-32

Christodoulou, C., Douzenis, A., Papadopoulos, F. C., Papadopoulou, A., Bouras, G., Gournellis, R., \& Lykouras, L. (2012). Suicide and seasonality. Acta Psychiatr Scand, 125(2), 127-46. doi:10.1111/j.1600-0447.2011.01750.x
Clarke, M., Moran, P., Keogh, F., Morris, M., Kinsella, A., Larkin, C., O'Callaghan, E. (1999). Seasonal influences on admissions for affective disorder and schizophrenia in Ireland: a comparison of first and readmissions. European Psychiatry : The Journal of the Association of European Psychiatrists, 14(5), 251-255.

CNRSSM. (2008). Relatório da Comissão Nacional para a Restruturação dos Serviços de Saúde Mental - Proposta de Plano de Ação para a Restruturação dos Serviços de Saúde Mental 2007-2016 (p. 156). Lisboa.

De Velde, S. Van, Boyd, A., \& Masfety, V. K. (2011). The Gender Perspective of Mental Health in Europe.

DGS. (2006). Risco de morrer em Portugal 2004 (p. 229). Lisboa: Direcção-Geral da Saúde.

DGS. (2013). Saúde Mental em números - 2013. Lisboa.

DGS. (2014). Saúde Mental em números - 2014. Lisboa.

Dixon, P., \& Kalkstein, A. (2009). Climate-suicide relationships: A research problem in need of geographic methods and cross-disciplinary perspectives. Geography Compass, 3(6), 1-14. doi:10.1111/j.1749-8198.2009.00286.x

Eaton, N., Keyes, K., Krueger, R., Balsis, S., Skodol, A. , Markon, K., Grant, B., Hasin, D. S. (2012). An invariant dimensional liability model of gender differences in mental disorder prevalence: evidence from a national sample. Journal of Abnormal Psychology, 121(1), 282288. doi:10.1037/a0024780

Eurowinter Group. (1997). Cold exposure and winter mortality from ischaemic heart disease, cerebrovascular disease, respiratory disease, and all causes in warm and cold regions of Europe. The Eurowinter Group. The Lancet, 349(9062), 1341-6. doi:10.1016/S01406736(96)12338-2

Giles-Sims, J., \& Lockhart, C. (2006). Explaining Cross-State Differences in Elderly Suicide Rates and Identifying State-Level Public Policy Responses that Reduce Rates. Suicide \& Life-Threatening Behavior, 36(6), 694-708. doi:10.1521/suli.2006.36.6.694

Hare, E., \& Walter, S. (1978). Seasonal variation in admissions of psychiatric patients and its relation to seasonal variation in their births. Journal of Epidemiology and Community Health, 32(1), 47-52.

Healy, J. (2003). Excess winter mortality in Europe: a cross country analysis identifying key risk factors. Journal of Epidemiology and Community Health, 57(10), 784-789. doi:10.1136/jech.57.10.784

Holley, H. (1998). Geography and mental health: a review. Social Psychiatry and Psychiatric Epidemiology, 33(11), 535-42. doi:10.1007/s001270050091 
Hsu, C., Chang, S., Lee, E., \& Yip, P. (2015). Geography of suicide in Hong Kong: Spatial patterning, and socioeconomic correlates and inequalities. Social Science \& Medicine, 130, 190-203. doi:10.1016/j.socscimed.2015.02.019

INE, \& INSA. (2015). Destaque: Informação à comunicação social. Inquérito Nacional de Saúde 2014. Lisboa.

Loureiro, A., Costa, C., Almendra, R., Freitas, Â. \& Santana, P. (2015). The socio-spatial context as a risk factor for hospitalization due to mental illness in the metropolitan areas of Portugal, Cadernos de Saúde Pública, 31, 219-231

Monteiro, A., \& Velhas, E. (1995). Estados de tempo e suicídio : coincidência ou consequência? Porto : Universidade do Porto.

Moriarty, D., Zack, M., Holt, J., Chapman, D., \& Safran, M. (2009). Geographic Patterns of Frequent Mental Distress. U.S. Adults, 1993-2001 and 2003-2006. American Journal of Preventive Medicine, 36(6), 497-505. doi:10.1016/j.amepre.2009.01.038

Nicolau, R., Machado, A., Falcão, J., \& Lira, M. (2009). Distribuição dos Internamentos Hospitalares em Portugal Continental: Agregação Geográfica e Determinantes (INSA). Lisboa.

Nogueira, P., Nunes, A., Nunes, B., Falcão, J., \& Ferrinho, P. (2009). Internamentos hospitalares associados à onda de calor de Agosto de 2003: evidências de associação entre morbilidade e ocorrência de calor. Revista Portuguesa de Saúde Pública, 27(2), 87-102.

Patel, V., Lund, C., Hatherill, S., Plagerson, S., Corrigall, J., Funk, M., \& Flisher, A. (2010). Mental disorders: equity and social determinants. In A. Blas \& A. S. Kurup (Eds.), Equity, Social Determinants and Public Health Programs (pp. 115-134). Genebra: World Health Organization.

Pinheiro, C. (1990). Um frio de morrer ou a variação da mortalidade e clima nos distritos de Viana do Castelo e de Faro. Arquivos Do Instituto Nacional de Saúde, (15), 61-112.

Rau, R. (2006). Seasonality in Human Mortality: A Demographic Approach. Berlin: Springer.

Roehner, B. (2015). How can one explain changes in the monthly pattern of suicide? Physica A: Statistical Mechanics and Its Applications, 424, 350-362. doi:10.1016/j.physa.2015.01.017

Rohrer, J., Rush Pierce, J., \& Blackburn, C. (2005). Lifestyle and mental health. Preventive Medicine, 40(4), 438-443. doi:10.1016/j.ypmed.2004.07.003

Santana, P. (2005). Geografias da Saúde e do Desenvolvimento - Evolução e Tendências em Portugal (p. 342). Coimbra: Almedina.
Santana, P., Costa, C., Cardoso, G., Loureiro, A., \& Ferrão, J. (2015). Suicide in Portugal: Spatial determinants in a context of economic crisis. Health \& Place, 35, 8594. doi:10.1016/j.healthplace.2015.07.001

Shapira, A., Shiloh, R., Potchter, O., Hermesh, H., Popper, M., \& Weizman, A. (2004). Admission rates of bipolar depressed patients increase during spring/summer and correlate with maximal environmental temperature. Bipolar Disorders, 6(1), 90-3.

Whiteford, H., Degenhardt, L., Rehm, J., Baxter, A., Ferrari, A., Erskine, H., Charlson, F., Norman, R., Flaxman, A., Johns, N., Brustein, R., Vos, T. (2013). Global burden of disease attributable to mental and substance use disorders: Findings from the Global Burden of Disease Study 2010. The Lancet, 382(9904), 1575-1586. doi:10.1016/S0140-6736(13)61611-6

Woo, J., Okusaga, O., \& Postolache, T. (2012). Seasonality of suicidal behavior. International Journal of Environmental Research and Public Health, 9(2), 531-47. doi:10.3390/ijerph9020531 